\title{
Controlling qubit transitions during non-adiabatic rapid passage through quantum interference
}

\author{
Frank Gaitan \\ Department of Physics \\ Southern Illinois University \\ Carbondale, IL 62901-4401
}

December 27, 2018

\begin{abstract}
In adiabatic rapid passage, the Bloch vector of a qubit is inverted by slowly inverting an external field to which it is coupled, and along which it is initially aligned. In non-adiabatic twisted rapid passage, the external field is allowed to twist around its initial direction with azimuthal angle $\phi(t)$ at the same time that it is non-adiabatically inverted. For polynomial twist, $\phi(t) \sim B t^{n}$. We show that for $n \geq 3$, multiple qubit resonances can occur during a single inversion of the external field, producing strong interference effects in the qubit transition probability. The character of the interference is controllable through variation of the twist strength $B$. Both constructive and destructive interference are possible, allowing qubit transitions to be greatly enhanced or suppressed. Experimental confirmation of these controllable interference effects has already occurred. Application of this interference mechanism to the construction of fast fault-tolerant quantum controlled-NOT and NOT gates is discussed.
\end{abstract}

\section{Introduction}

To set the stage for the work to be presented in this paper, we remind the reader of two fundamental results from the theory of quantum computation: (1) the existence of universal sets of quantum logic gates; and (2) the possibility of fault-tolerant quantum computation.

(1) In the quantum circuit model of quantum computation [1, a network of quantum logic gates is used to implement a desired quantum computation. An $n$-qubit quantum logic gate is a device that performs a fixed unitary transformation $U$ on the state of $n$ qubits. Just as with classical logic gates, universal sets of quantum logic gates have been shown to exist so that any quantum computation involving a finite number of qubits can be carried out using a network composed entirely of gates belonging to the universal set [1, 2, 3]. For example, the 1-qubit Hadamard gate, the 1-qubit phase gate, and the 2-qubit controlledNOT (CNOT) gate form a universal set of gates.

(2) It has been shown that a quantum computation of arbitrary duration using $n$ qubits can be carried out with arbitrarily small error probability if all gates used in the computation have an error probability (per gate operation) $P$ that falls below a threshold value $P_{a}$ known as the accuracy threshold [4. A quantum gate is said to operate fault-tolerantly if its error probability satisfies $P<P_{a}$. Estimates of $P_{a}$ have been made using different error models to describe the effect of the environment on the quantum gate. The best known estimate [5] considered a model in which the environment subjects a quantum gate to a classical stochastic process that generates independent errors. For this model, $P_{a} \sim 10^{-4}$. Other error models have yielded smaller values for $P_{a}$, although the value of $10^{-4}$ has become an unofficial benchmark for fault-tolerant operation of a quantum gate. Determining how to construct fast quantum gates that also operate fault-tolerantly $\left(P<10^{-4}\right)$ is one of the major technical challenges facing the quantum computing community. The work reported in this paper describes a promising approach for constructing fast fault-tolerant quantum NOT and CNOT gates which exploits quantum interference effects to control qubit transitions. Both of these gates appear often in quantum algorithms, with the CNOT gate being especially important for quantum error correction.

To round out this introduction, and to set notation, we briefly summarize the unitary transformations implemented by quantum NOT and CNOT gates. The quantum analogues of the classical bit states 0 and 1 are the 1-qubit computational ba- 
sis states (CBS) $|0\rangle$ and $|1\rangle$. Any pair of orthonormal basis states can serve as 1-qubit CBS. A quantum NOT gate transforms $|0\rangle \longleftrightarrow|1\rangle$ so that its action on the 1-qubit CBS is $U_{\text {NOT }}|i\rangle=|i \oplus 1\rangle$. Here $\oplus$ denotes addition modulo 2 , and $i=0,1$. Since $U_{\text {NOT }}$ is a linear operator, its action on an arbitrary 1-qubit state follows from its action on the CBS: $U_{\text {NOT }}(a|0\rangle+b|1\rangle)=a|1\rangle+b|0\rangle$. The quantum CNOT gate is a 2 -qubit gate. The 2-qubit CBS $|i j\rangle$ are obtained by forming all possible tensor products of the 1-qubit CBS: $|i j\rangle=|i\rangle_{c} \otimes|j\rangle_{t}$, with $i, j=0,1$. The $c(t)$ subscript indicates that the first (second) ket corresponds to the control (target) qubit. The action of a quantum CNOT gate on the 2-qubit CBS is $U_{C N O T}|i j\rangle=|i\rangle_{c} \otimes|j \oplus i\rangle_{t}$. Thus $U_{C N O T}$ applies a NOT operation to the target qubit only when the control qubit has $i=1$. The action of $U_{C N O T}$ on an arbitrary 2-qubit state follows from linearity.

The structure of this paper is as follows. In the next section we examine 3 ways of implementing a quantum NOT gate. The first two are well-known and are discussed to show what is possible with familiar technology. The third approach uses a less familiar form of rapid passage known as non-adiabatic twisted rapid passage [6]. We show that during this type of rapid passage the qubit can pass through resonance multiple times during a single rapid passage sweep, and that quantum interference effects are generated that allow strong control of qubit transitions. It is important to note that these quantum interference effects have recently been observed using liquid state NMR [7. We close Section 2 by showing that a fast fault-tolerant quantum NOT gate based on twisted rapid passage is possible with existing NMR technology. In section [3 we show how a quantum CNOT gate can be implemented using twisted rapid passage, and we summarize and make final remarks in Section 4

\section{Quantum NOT gate: 3 ways}

\subsection{Adiabatic rapid passage}

Adiabatic rapid passage (ARP) is a well-known procedure that inverts the Bloch vector of a qubit by inverting an external field $\mathbf{F}(t)$ to which it is coupled, and along which it was initially aligned. The qubit is coupled to $\mathbf{F}(t)$ through the Zeeman interaction,

$$
H(t)=-\boldsymbol{\sigma} \cdot \mathbf{F}(t),
$$

and the external field is inverted such that $\mathbf{F}(t)=$ $b \hat{\mathbf{x}}+a t \hat{\mathbf{z}}$. The instantaneous energy eigenvalues of $H(t)$ are $E_{ \pm}(t)= \pm \sqrt{b^{2}+(a t)^{2}}$, and we denote the instantaneous energy eigenstates by $\left|E_{ \pm}(t)\right\rangle$. From eq. (1) we see that the Bloch vector $\langle\boldsymbol{\sigma}\rangle_{t}$ is parallel (anti-parallel) to $\hat{\mathbf{F}}(t)$ in the eigenstate $\left|E_{-}(t)\right\rangle$ $\left(\left|E_{+}(t)\right\rangle\right)$. An avoided crossing occurs at $t=0$ where the energy gap is smallest, and one can show that the qubit is at resonance at this crossing (see Section [2.3.3). Without loss of generality, the inversion can be considered to take place during the time interval $[-T / 2, T / 2]$. For ARP, the inversion time $T$ is much larger than the inverse Rabi frequency (viz. adiabatic), yet short compared to the thermal relaxation time $\tau$ (viz. rapid). One also has that $a T \gg b$ so that $\hat{\mathbf{F}}(t)$ is effectively aligned with $\pm \hat{\mathbf{z}}$ as $t \rightarrow \pm T / 2$. The qubit is initially prepared in an energy eigenstate of the initial Hamiltonian with it's Bloch vector initially pointing along, say, $-\hat{\mathbf{z}}$. Then $|\psi(-T / 2)\rangle=\left|E_{-}(-T / 2)\right\rangle$. Expanding $|\psi(t)\rangle$ in terms of the basis states $\left|E_{ \pm}(t)\right\rangle$ gives,

$$
|\psi(t)\rangle=S(t)\left|E_{-}(t)\right\rangle+I(t)\left|E_{+}(t)\right\rangle .
$$

$S(t)$ is the probability amplitude that the qubit will be found in the $E_{-}$energy-level at time $t$, and $I(t)$ is the probability amplitude that a transition has occurred, and that the qubit will be found in the $E_{+}$energy-level at time $t$. The Schrodinger dynamics for ARP can be solved exactly for arbitrary values of $a$ and $b$ [ 8 . The final transition probability $P=|I(T / 2)|^{2}$ is given by the Landau-Zener expression,

$$
P=\exp [-\pi / \lambda],
$$

where $\lambda=\hbar a / b^{2}$ (and we are assuming that $a, b>$ $0)$. For ARP, $\lambda \ll 1$ so that the transition probability is exponentially small. Thus transitions can be safely ignored during ARP, and to an excellent approximation, we can write:

$$
|\psi(t)\rangle=\left|E_{-}(t)\right\rangle .
$$

Eq. (41) indicates that the qubit Bloch vector remains parallel to $\mathbf{F}(t)$ throughout its inversion. Thus ARP causes the qubit Bloch vector to be inverted as a consequence of the inversion of the external field $\mathbf{F}(t)$.

If we define the CBS so that $|0\rangle=\left|\sigma_{z}=-1\right\rangle=$ $\left|E_{-}(-T / 2)\right\rangle$ and $|1\rangle=\left|\sigma_{z}=+1\right\rangle=\left|E_{-}(+T / 2)\right\rangle$, the previous remarks indicate that ARP causes $|0\rangle \longleftrightarrow$ $|1\rangle$. Thus ARP implements a quantum NOT operation on the qubit. Note that a transition during ARP means that $\left|E_{ \pm}(-T / 2)\right\rangle \rightarrow\left|E_{\mp}(+T / 2)\right\rangle$, or in terms of the CBS: $|i\rangle \rightarrow|i\rangle$. The occurrence of a transition during ARP thus corresponds to an error in the quantum NOT operation. The Landau-Zener expression (eq. (3) ) for the transition probability $P$ thus gives the error probability (per gate operation) for an ARP quantum NOT gate. This error probability can be 
made arbitrarily small by making the inversion take place at a sufficiently adiabatically rate. Thus, ARP can be used to implement a fault-tolerant, though adiabatically slow, quantum NOT gate.

\section{$2.2 \pi$-Pulse}

In a $\pi$-pulse, one inverts the qubit Bloch vector through application of a pulsed external field $\mathbf{F}(t)=$ $F(t) \hat{\mathbf{e}}$ whose direction, power, and duration are chosen to insure that the Bloch vector undergoes a $180^{\circ}$ rotation about $\hat{\text { e. }}$. In the usual situation, the qubit initial state $|\psi(-T / 2)\rangle$ is an eigenstate of $\sigma_{z}$, and the external field has $\hat{\mathbf{e}}=\hat{\mathbf{x}}$. If we write $\mid \sigma_{z}=$ $-1\rangle=|\downarrow\rangle$ and $\left|\sigma_{z}=+1\right\rangle=|\uparrow\rangle$, then a $\pi$-pulse maps $|\downarrow\rangle \longleftrightarrow|\uparrow\rangle$. Defining the 1-qubit CBS as $|0\rangle=|\downarrow\rangle$ and $|1\rangle=|\uparrow\rangle$, we see that a $\pi$-pulse implements a quantum NOT gate: $|0\rangle \longleftrightarrow|1\rangle$. Since the pulse power, duration, and direction cannot be perfectly controlled, a real $\pi$-pulse will execute an imperfect quantum NOT operation. Fortunato et. al. 9] have worked with non-adiabatic $\pi$-pulses (in NMR) whose error probability (per NOT operation) satisfies $P>3 \times 10^{-4}$. This is only slightly larger than the benchmark value for the accuracy threshold of $10^{-4}$. If we denote the pulse amplitude by $F_{1}$, then for $\omega_{1}=\gamma F_{1} \sim 4000 \mathrm{~Hz}(\gamma=$ gyromagnetic ratio), the pulse duration will be $T=\pi / \omega_{1} \sim 1$ msec. Thus the best $\pi$-pulses can implement fast, though not quite fault-tolerant, quantum NOT gates.

\subsection{Twisted rapid passage}

Twisted rapid passage (TRP) generalizes ARP in two essential ways: (1) the adiabatic restriction is relaxed; and (2) the external field $\mathbf{F}(t)$ is allowed to twist around its initial direction during the course of its inversion. Specifically, the time dependence of the external field during TRP is: $\mathbf{F}(t)=b \cos \phi(t) \hat{\mathbf{x}}+$ $b \sin \phi(t) \hat{\mathbf{y}}+a t \hat{\mathbf{z}}$. In this subsection we show that multiple qubit resonances can occur per TRP sweep, and that by varying their time separation, quantum interferences effects are produced which allow for a direct control over qubit transitions. We then discuss the experimental confirmation of this interference mechanism for controlling qubit transitions, and show how TRP can be used to implement a fast faulttolerant quantum NOT gate. A detail presentation of these results is given in refs. [6] and [7].

\subsubsection{Multiple resonances}

It proves convenient to transform to the rotating frame in which the $x-y$ component of the external field is instantaneously at rest. This is ac- complished via the unitary transformation $U(t)=$ $\exp \left[-(i / 2) \phi(t) \sigma_{z}\right]$. The Hamiltonian $\bar{H}(t)$ in this frame is

$$
\bar{H}(t)=-\boldsymbol{\sigma} \cdot \overline{\mathbf{F}}(t),
$$

and $\overline{\mathbf{F}}(t)=b \hat{\mathbf{x}}+(a t-\hbar \dot{\phi} / 2) \hat{\mathbf{z}}$ is the external field as seen in the rotating frame, and a dot over a symbol represents the time derivative of that symbol. The instantaneous energy eigenvalues are $\bar{E}_{ \pm}(t)=$ $\pm \sqrt{b^{2}+(a t-(\hbar \dot{\phi} / 2))^{2}}$. Avoided crossings occur when the energy gap is minimum, corresponding to when

$$
a t-\frac{\hbar}{2} \frac{d \phi}{d t}=0 .
$$

In Section 2.3.3 we show that qubit resonance occurs at an avoided crossing. For polynomial twist, $\phi_{n}(t)=c_{n} B t^{n}$, where $B$ is the twist strength. The dimensionless constant $c_{n}$ has been introduced to simplify some of the formulas below. For later convenience we choose $c_{n}=2 / n$. For polynomial twist it is easily checked that eq. (6) always has the root

$$
t=0
$$

and that for $n \geq 3$, eq. (6) also has the $n-2$ roots,

$$
t=(\operatorname{sgn} B)^{\frac{1}{(n-2)}}\left(\frac{a}{\hbar|B|}\right)^{\frac{1}{(n-2)}} .
$$

All together, eq. (6) has $n-1$ roots, though only the real roots correspond to qubit resonances. For quadratic twist $(n=2)$, only eq. (7) arises. For $n \geq 3$, however, along with the resonance at $t=0$, real solutions to eq. (8) also occur. The various possibilities for this situation are summarized in Table1.

\begin{tabular}{l}
\hline (1) $\frac{\operatorname{sgn} \mathrm{B}=+1}{n \text { odd; resonances at: } t=0 \text { and }(a / \hbar B)^{\frac{1}{(n-2)}}}$ \\
$n$ even; resonances at: $t=0$ and $\pm(a / \hbar B)^{\frac{1}{(n-2)}}$ \\
$(2) \underline{\operatorname{sgn} \mathrm{B}=-1}$ \\
$n$ odd; resonances at: $t=0$ and $-(a / \hbar|B|)^{\frac{1}{(n-2)}}$ \\
$n$ even; resonance at: $t=0$
\end{tabular}

Table 1: Classification of regimes under which multiple qubit resonances occur for polynomial twist with $n \geq 3$.

We see that for polynomial twist with $n \geq 3$, multiple qubit resonances always occur per TRP sweep 
for positive twist strength $B$, while for negative twist strength, multiple resonances only occur when $n$ is odd. It is important to note that the time separating the multiple qubit resonances can be varied through a variation of the twist strength $B$ and/or the inversion rate $a$ (see eq. (8)).

\subsubsection{Controllable quantum interferences}

To determine the dynamical impact of TRP we simulated the qubit Schrodinger equation numerically in the non-rotating frame. The details of this simulation are described in ref. [6]. The equations governing the time evolution of the probability amplitudes $S(t)$ and $I(t)$ (see eq. (2)) are easily obtained from the Schrodinger equation and it is these equations that are numerically integrated. It proves convenient to re-write these equations in dimensionless form. To that end, one introduces the dimensionless time $\tau=(a / b) t$, the dimensionless inversion rate $\lambda=\hbar|a| / b^{2}$, and the dimensionless twist strength $\eta_{n}$,

$$
\eta_{n}=\frac{\hbar B}{a}\left(\frac{b}{a}\right)^{n-2} .
$$

From eqs. (7) and (8), the (dimensionless) times at which the multiple resonances occur are,

$$
\tau=0
$$

and

$$
\tau=\left(\operatorname{sgn} \eta_{n}\right)^{\frac{1}{(n-2)}}\left[\frac{1}{\left|\eta_{n}\right|}\right]^{\frac{1}{(n-2)}} .
$$

Only the real solutions of eq. (11) correspond to qubit resonances. Ref. [6] examined cubic $(n=3)$ and quartic $(n=4) \mathrm{TRP}$ in detail. These cases correspond to the simplest examples of odd and even order twist, respectively, that contain multiple qubit resonances. Due to space limitations, we only review the results for quartic twist in this paper. The reader is referred to ref. 6] for the cubic TRP results. For quartic twist $\phi_{4}(t)=(1 / 2) B t^{4}$ and $\eta_{4}=\hbar B b^{2} / a^{3}$. The analysis of Section 2.3.1 indicates that qubit resonances will occur at $\tau=0$ and $\tau= \pm 1 / \sqrt{\eta_{4}}$ when $\operatorname{sgn} \eta_{4}=+1$, and only at $\tau=0$ when $\operatorname{sgn} \eta_{4}=-1$. The initial condition for the simulation is $\left|\psi\left(-\tau_{0} / 2\right)\right\rangle=\left|E_{-}\left(-\tau_{0} / 2\right)\right\rangle$, and $\tau_{0}=(a / b) T$. We will be interested in the transition probability $P(t)=|I(t)|^{2}$.

For purposes of comparison, Figure 11 shows the transition probability $P(\tau)$ vs. $\tau$ for twistless rapid passage with $\lambda=5.0$ (non-adiabatic) and $\eta_{4}=0$. The final transition probability $P$ at $\tau_{0} / 2$ was found to be $P=0.533$. Figure 2 plots the transition probability $P(\tau)$ for $\lambda=5.0$ and $\eta_{4}=4.6 \times 10^{-4}$. The

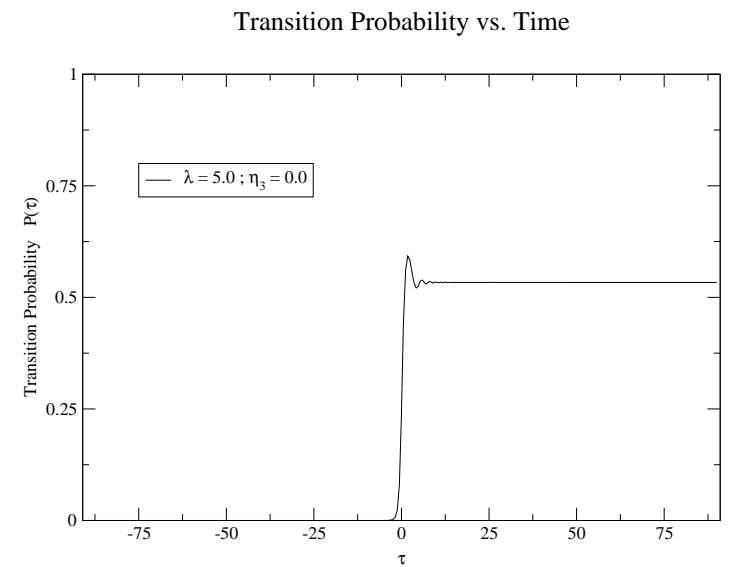

Figure 1: Plot of the transition probability $P(\tau)$ for twistless non-adiabatic rapid passage with $\lambda=5.0$ and $\eta=0$.

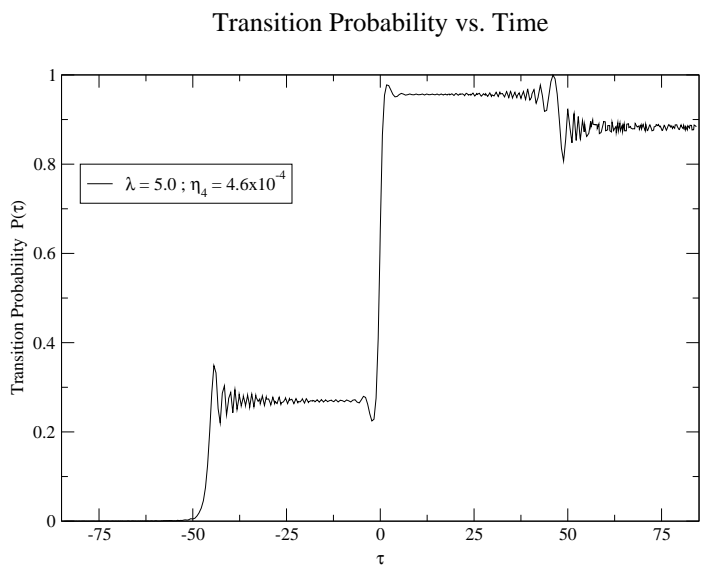

Figure 2: The transition probability $P(\tau)$ for nonadiabatic rapid passage with quartic twist with $\lambda=$ 5.0 and $\eta_{4}=4.6 \times 10^{-4}$.

expected qubit resonances at $\tau=0$ and $\tau= \pm 46.63$ are clearly visible. The final transition probability for this case is $P=0.88$. As we have just seen, twistless rapid passage with $\lambda=5.0$ has $P=0.533$. Thus the resonances in Figure 2 are constructively interfering, leading to an enhancement of the qubit transition probability $P$. Figure 3 shows $P(\tau)$ for quartic twist with $\lambda=5.0$ and $\eta_{4}=-4.6 \times 10^{-4}$. This figure clearly shows only one resonance at $\tau=0$, as expected for $\operatorname{sgn} \eta_{4}=-1$ (see Table 1). The final transition probability for this case is $P=0.533$, which equals the result for twistless rapid passage with $\lambda=5.0$, as one might expect given the presence of only one qubit resonance in both cases.

Figure 4 plots $P(\tau)$ for $\lambda=5.0$ and $\eta_{4}=1.6 \times 10^{-3}$. The figure clearly shows the expected resonances at $\tau=0$ and $\tau= \pm 25.0$. The final transition probabil- 
Transition Probability vs. Time

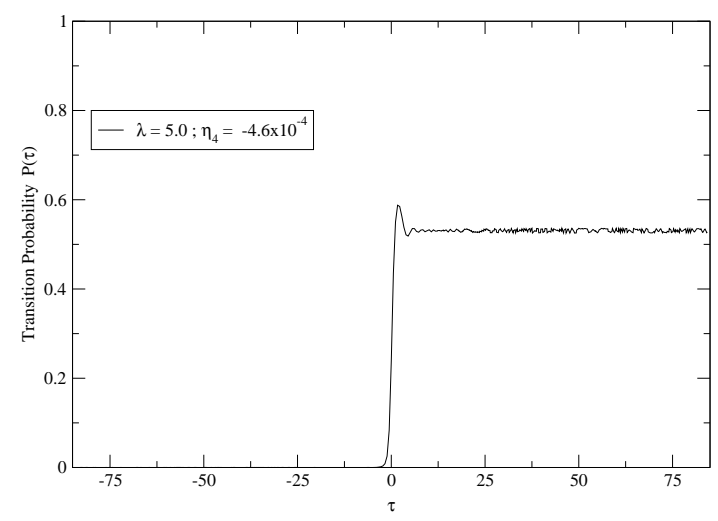

Figure 3: The transition probability $P(\tau)$ for nonadiabatic rapid passage with quartic twist with $\lambda=$ 5.0 and $\eta_{4}=-4.6 \times 10^{-4}$.

Transition Probability vs. Time

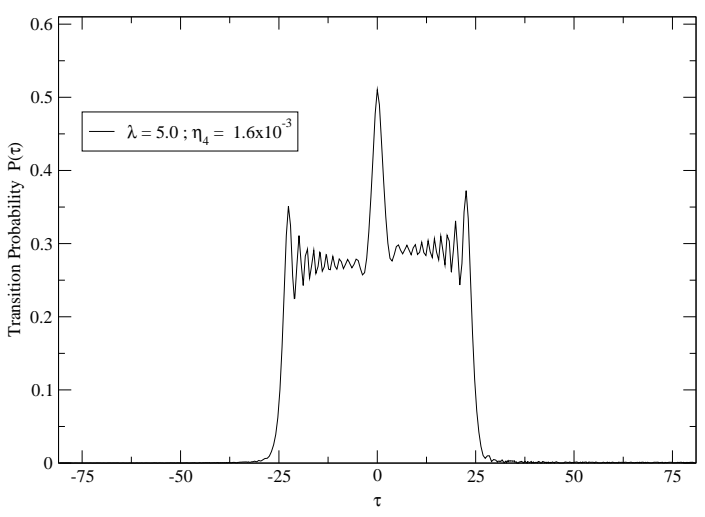

Figure 4: The transition probability $P(\tau)$ for nonadiabatic rapid passage with quartic twist with $\lambda=$ 5.0 and $\eta_{4}=1.6 \times 10^{-3}$. Note the slightly reduced vertical scale.

ity is $P=6.93 \times 10^{-4}$, corresponding to destructive interference relative to twistless rapid passage with $\lambda=5.0$. Note that adding a small amount of quartic twist has lowered the final transition probability $P$ by three orders-of-magnitude. We do not include a plot of $P(\tau)$ for $\lambda=5.0$ and $\eta_{4}=-1.6 \times 10^{-3}$ as it is similar to Figure 3 namely one resonance at $\tau=0$ and $P=0.533$.

Summarizing these results, we see that: (i) three (one) qubit resonances (resonance) occur(s) as predicted in Table 1 when $\operatorname{sgn} \eta_{4}=+1(-1)$; (ii) the qubit resonances produce strong interference effects in the qubit transition probability, with the character of the interference (constructive or destructive) determined by the time separation of the resonances; and (iii) the time separation of adjacent qubit reso- nances is given by $\Delta \tau=1 / \sqrt{\eta_{4}}\left(\operatorname{sgn} \eta_{4}=+1\right)$, and can be controlled through variation of $\eta_{4}=\hbar B b^{2} / a^{3}$.

\subsubsection{Experimental realization}

As pointed out in the Introduction, these multiresonance induced quantum interference effects have been experimentally confirmed by Zwanziger et. al. using liquid state NMR 7. Both cubic and quartic twist were experimentally realized. In the experiment a driving rf field is linearly polarized along the $x$ axis in the lab frame with $F_{x}(t)=2 b \cos \phi_{r f}(t)$. The resonance offset at is produced by linearly sweeping the detector frequency $\omega_{\text {det }}(t)$ through resonance at the Larmor frequency $\omega_{0}$ such that $\omega_{\text {det }}(t)=\omega_{0}+$ $(2 a t / \hbar)$. Twist is introduced by sweeping the rf frequency $\omega_{r f}(t)=\dot{\phi}_{r f}$ through resonance at $\omega_{0}$ in such a way that $\omega_{r f}(t)=\omega_{d e t}-\dot{\phi}_{n}$, and $\phi_{n}(t)=(2 / n) B t^{n}$ is the azimuthal angle for twisted rapid passage introduced in Section 2.3.1 Note that the resonance condition $\omega_{r f}(t)=\omega_{0}$ is identical to our existence condition for an avoided crossing, eq. (6). This establishes the promised correspondence between avoided crossings and qubit resonances. The comparison between experiment and theory is given in Figure 5 for cubic twist, and Figure 6] for quartic twist. We see

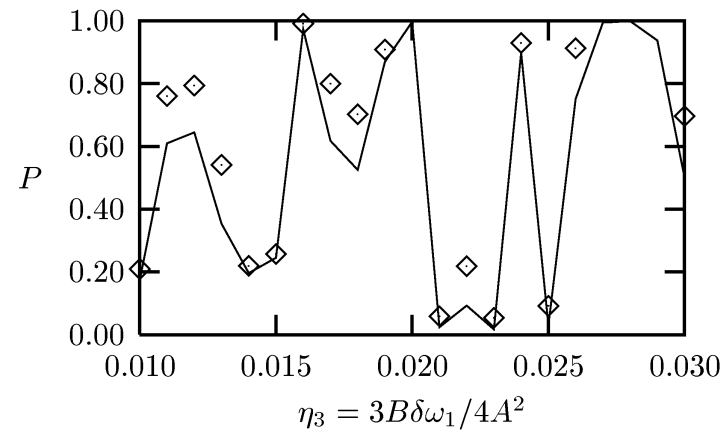

Figure 5: Data and simulation for a cubic sweep profile, as a function of the dimensionless parameter $\eta_{3}=3 B \delta \omega_{1} / 4 A^{2}$. In the data shown, $A=50,000$ $\mathrm{Hz}, \delta=24.39 \mathrm{~Hz}, \omega_{1}=393 \mathrm{~Hz}$, and $B$ is calculated from the target $\eta_{3}$.

that: (1) variation of the twist strength $\eta_{4}$ clearly causes the qubit transition probability $P$ to move between constructive and destructive interference; and (2) the agreement between theory and experiment is excellent. We refer the reader to ref. [7] and [10] for a detailed discussion of the experimental parameters, and to the Appendix of ref. 6] for the translation key that connects our theoretical parameters to the 


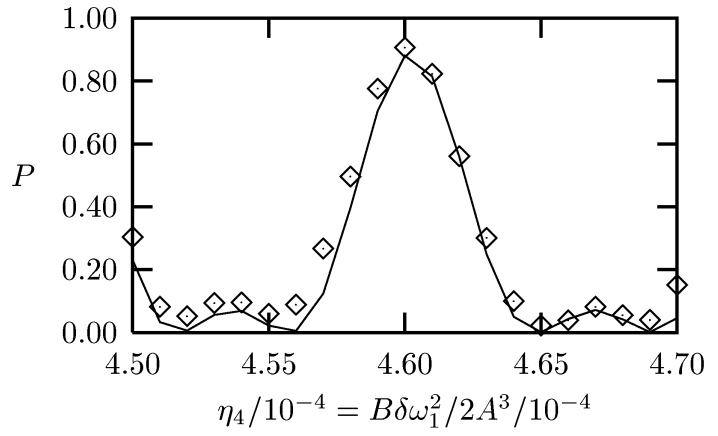

Figure 6: Data and simulation for a quartic sweep profile, as a function of the dimensionless parameter $\eta_{4}=B \delta \omega_{1}^{2} / 2 A^{3}$. In the data shown, $A=50,000 \mathrm{~Hz}$, $\delta=24.39 \mathrm{~Hz}, \omega_{1}=393 \mathrm{~Hz}$, and $B$ is calculated from the target $\eta_{4}$.

experimental parameters of the Zwanziger et. al. experiments.

Before leaving the subject of experimental realization of twisted rapid passage, two further remarks are in order. First, to insure that all qubits are inverted when a spread of resonance frequencies occurs, it is necessary to require that the frequency sweep cover a large enough interval that the entire spread of resonance frequencies is included in it. This gaurantees that all qubits will have passed through resonance by the end of the frequency sweep. Second, a range of rf field strengths can also be accommodated so long as $a T / 2 \gg b_{\max }$. This condition insures that the frequency sweep begins far from resonance for all rf field strengths, and that transitions will continue to occur only near the avoided crossings. One therefore anticipates that in this case also, the interference effects will continue to occur as predicted. For reasonably good samples, magnets, and rf sources, these constraints can be satisfied, and the interference effects presented above should be readily observable. This is in fact what is found experimentally 7 .

\subsubsection{Quantum NOT gate}

We restrict our attention here to quartic twist, though our discussion is readily modified to treat other cases of TRP. As seen in Section 2.3.1 the external field $\overline{\mathbf{F}}(t)$ seen in the rotating frame has $z$ component $\bar{F}_{z}(t)$ which (written in terms of dimensionless time $\tau=a t / b)$ is $\bar{F}_{z}(\tau)=b \tau\left(1-\eta_{4} \tau^{2}\right)$. Thus the direction of $\overline{\mathbf{F}}(t)$ approaches $\mp \hat{\mathbf{z}}$ as $t \rightarrow$ $\pm T / 2$. This asymptotic behavior allows us to implement a quantum NOT gate using quartic twist in a manner that parallels the ARP approach dis-

\begin{tabular}{cc}
\hline \hline$\eta_{4}\left(\times 10^{-3}\right)$ & $\mathrm{P}$ \\
\hline 3.95 & $2.0 \times 10^{-2}$ \\
3.96 & $1.3 \times 10^{-2}$ \\
3.97 & $6.8 \times 10^{-3}$ \\
3.98 & $3.6 \times 10^{-3}$ \\
3.99 & $9 \times 10^{-4}$ \\
4.00 & $4 \times 10^{-5}$ \\
4.01 & $8 \times 10^{-4}$ \\
4.02 & $3.9 \times 10^{-3}$ \\
4.03 & $1.0 \times 10^{-2}$ \\
4.04 & $1.7 \times 10^{-2}$ \\
\hline
\end{tabular}

Table 2: Transition probabilities for quartic twist with $\lambda=5.0$ and $\eta_{4}$ in the range $(3.95,4.04) \times 10^{-3}$.

cussed in Section [2.1] If we initially prepare the qubit in the $E_{-}$energy-level, and we define the CBS such that $|0\rangle=\left|\sigma_{z}=-1\right\rangle$ and $|1\rangle=\left|\sigma_{z}=+1\right\rangle$, then $|\psi(-T / 2)\rangle=\left|E_{-}(-T / 2)\right\rangle=|0\rangle$. In the absence of transitions, $|\psi(T / 2)\rangle=\left|E_{-}(T / 2)\right\rangle=|1\rangle$ and TRP with quartic twist thus implements a quantum NOT operation $|0\rangle \longleftrightarrow|1\rangle$. If a transition occurs, $\left|E_{ \pm}(-T / 2)\right\rangle \rightarrow\left|E_{\mp}(T / 2)\right\rangle$, or $|i\rangle \rightarrow|i\rangle$, corresponding to an error in the NOT operation. As with ARP, the transition probability $P=|I(T / 2)|^{2}$ gives the error probability (per gate operation) of the TRP quantum NOT gate. Table 2 gives the transition probabilities for quartic twist pulses for which $\lambda=5.0$ and $\eta_{4}$ lies in the interval $[3.95,4.04] \times 10^{-3}$. The essential thing to notice about Table 2 is that for $\eta_{4}=4.00 \times 10^{-3}$, the transition/error probability is $P=4 \times 10^{-5}$. This is less than the benchmark value for fault-tolerant operation of $10^{-4}$ ! Thus our quartic twist quantum NOT gate is able to operate faulttolerantly. We now show that for pulse parameters which can be realized with existing NMR technology, the inversion time for TRP matches that of a comparable $\pi$-pulse. As shown in ref. [6, the inversion time for quartic twist is given by,

$$
T_{4}=\frac{4 A}{\omega_{1}^{2} \lambda},
$$

where $\omega_{1}$ is related to the amplitude of the NMR rf signal, and $A$ is related to the TRP inversion rate (see refs. [6] and [7]). Current NMR technology can generate $\omega_{1}=4000 \mathrm{~Hz}$ and $A=40,000 \mathrm{~Hz}$. With $\lambda=5.0$, eq. (12) gives $T_{4}=2$ msec. We saw in Section 2.2 that a comparable $\pi$-pulse has an inversion time $T_{\pi} \sim 1$ msec, and an error probability $P_{\pi}>3 \times 10^{-4}$. Thus a quartic twist quantum NOT gate can match the inversion speed of a $\pi$-pulse, while delivering an orderof-magnitude smaller error probability. Specifically, quartic twist promises to deliver a fast fault-tolerant 
quantum NOT operation with existing NMR technology. This is a claim that $\pi$-pulses are currently unable to make. We note that Zwanziger et. al. implemented this particular case of quartic twist, but they were unable to resolve a transition probability as small as $P=4 \times 10^{-5}$ from a value of zero [11. Thus, a quantitative test of this prediction remains an open experimental challenge.

\section{TRP quantum CNOT gate}

We now describe a procedure for implementing a quantum CNOT gate using TRP in the context of liquid state NMR. If the liquid has low viscosity, one can ignore dipolar coupling between the qubits, and if the remaining Heisenberg interaction between the qubits is weak compared to the individual qubit Zeeman energies, it can be well-approximated by an Ising interaction [12]. Under these conditions, the Hamiltonian (in frequency units) for the control (c) and target $(t)$ qubits is

$$
\frac{H_{c t}}{\hbar}=-\omega_{c} I_{z}^{c}-\omega_{t} I_{z}^{t}+2 \pi J I_{z}^{c} I_{z}^{t}
$$

Here $\omega_{c}\left(\omega_{t}\right)$ is the resonance frequency of the isolated control (target) qubit, $J$ is the Ising coupling constant, and $\omega_{c}>\omega_{t}>\pi J$. We choose the singlequbit CBS to be the eigenstates of $\sigma_{z}$ with $|0\rangle=|\uparrow\rangle$ and $|1\rangle=|\downarrow\rangle$. Then the 2-qubit CBS are $|00\rangle=|\uparrow \uparrow\rangle$, $|01\rangle=|\uparrow \downarrow\rangle,|10\rangle=|\downarrow \uparrow\rangle$, and $|11\rangle=|\downarrow \downarrow\rangle$, and they are the eigenstates of $H_{c t}$. The energy levels (in frequency units) are shown in Figure 7 where

$$
\omega_{ \pm}=\omega_{t} \pm \pi J
$$
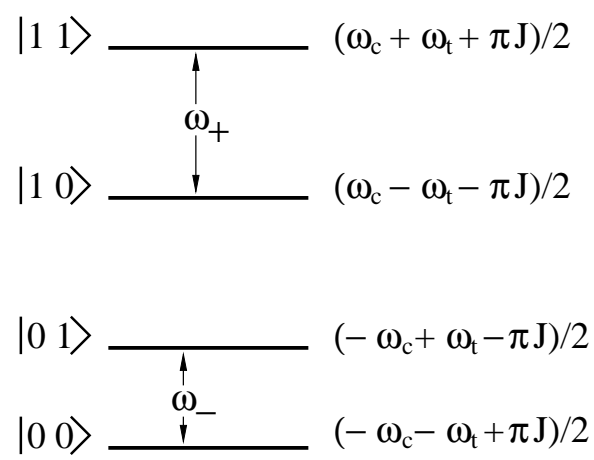

Figure 7: Energy-level structure appropriate for implementing a quantum CNOT operation using twisted rapid passage. The corresponding energies (in frequency units) appear to the right of the energylevels.
Given this energy-level structure, we can implement a quantum CNOT operation on the two qubits by sweeping through the $\omega_{+}$resonance using twisted rapid passage. Refocusing [13] is used to switch off the dynamics of the control qubit so that only the target qubit responds to the TRP pulse. Let $U$ denote the unitary transformation associated with this procedure. It maps the 2-qubit input state $\left|\psi_{\text {in }}\right\rangle$ at the beginning of the procedure to the output state $\left|\psi_{\text {out }}\right\rangle:\left|\psi_{\text {out }}\right\rangle=U\left|\psi_{\text {in }}\right\rangle$. Since the two states $|00\rangle$ and $|01\rangle$ are not resonant, they do not respond to the TRP pulse. Thus $U|00\rangle=|00\rangle$, and $U|01\rangle=|01\rangle$. On the other hand, for the $|10\rangle$ and $|11\rangle$ states, the combination of refocusing and sweeping through the $\omega_{+}$resonance means that only the target qubit has its spin inverted. Thus $U|10\rangle=|11\rangle$ and $U|11\rangle=|10\rangle$. This gives the action of $U$ on the 2-qubit CBS so that we can write out $U$ in the computational basis:

$$
U=\left(\begin{array}{llll}
1 & 0 & 0 & 0 \\
0 & 1 & 0 & 0 \\
0 & 0 & 0 & 1 \\
0 & 0 & 1 & 0
\end{array}\right)
$$

The right-hand side of eq. (15) is recognized as the unitary transformation implemented by a quantum CNOT gate [13], confirming that our procedure does in fact implement this gate on the two qubits. Knowing how $U$ acts on the 2-qubit CBS, linearity then determines its action on an arbitrary 2-qubit state.

\section{Discussion}

In this paper it has been our aim to show that multiple qubit resonances can occur during a single twisted rapid passage sweep, and that by varying their time separation, quantum interference effects are produced which allow for a direct control over qubit transitions. This time separation is controlled through the (dimensionless) twist strength $\eta$ (Section [2.3), and the resulting interference can be constructive (enhancing transitions) or destructive (suppressing transitions). These controllable interference effects are a consequence of the temporal phase coherence of the qubit wave function, and were experimentally confirmed using liquid state NMR by Zwanziger et. al. [7]. Cubic and quartic twist were considered in detail in ref. 6] (space limitations restricted our discussion here to quartic twist) as they are the simplest examples, respectively, of odd-order and even-order polynomial twist in which these interference effects are expected to occur. By focusing on these two examples we do not mean to suggest that these sweep profiles are the best of all possible twisted rapid passage profiles. A 
search is currently underway for other profiles that might produce stronger destructive interference effects. We have also shown how twisted rapid passage can be used to construct quantum NOT and CNOT gates. It was shown that parameter values for quartic twist exist that are realizable with current NMR technology and that will drive a fast fault-tolerant quantum NOT operation. This feat is currently beyond the capabilities of $\pi$-pulse and adiabatic rapid passage quantum NOT gates. Other work currently underway includes the following. (1) Development of an analytical scheme for approximately calculating the qubit transition probability. The aim here being to find trial sweep parameter values that will yield gate error probabilities $P<10^{-4}$. These trial values then serve as the starting point for a more focused numerical search that will yield the actual parameter values that will drive fast fault-tolerant quantum NOT and CNOT operations. (2) We are also working to resolve a technical complication associated with the resonance offset that arises with the quartic twist quantum CNOT gate (see 7] for further discussion).

\section{ACKNOWLEDGMENTS}

I would like to thank T. Howell III for continued support, the National Science Foundation for support provided through Grant No. NSF-PHY-0112335, and the Army Research Office for support provided through Grant No. DAAD19-02-1-0051.

\section{References}

[1] Deutsch, D., 1989, Proc. R. Soc. Lond. A 425, 73.

[2] DiVincenzo, D. P., 1995, Phys. Rev. A 51, 1015.

[3] Lloyd, S., 1995, Phys. Rev. Lett. 75, 346; Deutsch, D., Barenco, A., and Ekert, A., 1995, Proc. R. Soc. Lond. A 449, 669; Barenco, A., 1995, Proc. R. Soc. Lond. A 449, 679; Sleator, T., and Weinfurter, H., 1995, Phys. Rev. Lett. 74, 4087.

[4] For reviews see: Gottesman, D., 1997, Ph. D thesis, California Institute of Technology, http://arXiv.org/quant-ph/9705052 Preskill, J., 1998, Proc. R. Soc. Lond. A 454, 385; Knill, E., Laflamme, R., and Zurek, W. H., 1998, Science 279, 342 .

[5] See Gottesman, D. in reference 4].

[6] Gaitan, F., 2003, Phys. Rev. A 68, 052314.
[7] Zwanziger, J. W., Werner-Zwanziger, U., Gaitan, F., 2003, Chem. Phys. Lett. 375, 429.

[8] Landau, L., 1932, Phys. Z. Sowjetunion 1, 46; Zener, C., Proc. R. Soc. Lond. A 137, 696.

[9] Fortunato, E. M., Pravia, M. A., Boulant, N., Teklemariam, G., Havel, T. F., Cory, D. G., 2002, J. Chem. Phys. 116, 7599.

[10] Zwanziger, J. W., Rucker, S. P., Chingas, G. C., 1991, Phys. Rev. A 43, 3232.

[11] Zwanziger, J. W., private communication.

[12] Gershenfeld, N. A., and Chuang, I. L., 1997, Science 275, 350 .

[13] Chuang, I. L., 1998, in Introduction to Quantum Computation and Information, edited by H. K. Lo, S. Popescu, and T. Spiller (World Scientific, Teaneck, NJ). 